\title{
Modèle d'écoulement dans les bâches fronto-spirales
}

\section{A model of the flow in frontospiral casing}

\author{
J. Corniglion, P. Vinh et M. Couston
}

Société Neyrpic Grenoble

\section{Introduction}

La bâche fronto-spirale est un organe très important d'une turbine Kaplan. En effet, associée à des avantdirectrices (AV. D.) elle conditionne l'écoulement dans les organes situés à l'aval, donc outre les pertes d'énergie qu'elle occasionne, elle est responsable d'une partie des pertes que l'on impute aux directrices. Si l'on considère que les pertes de l'amenée sont de l'ordre de 20 à $30 \%$ des pertes totales au cœur de la colline, aussi bien qu'à forte charge on voit tout l'intérêt qu'il y a à bien maîtriser la conception de la bâche.

Depuis plusieurs années, Neyrpic a décidé, non sans succès, de faire porter une partie des recherches Kaplan sur la conception des bâches fronto-spirales. En ce qui concerne l'approche théorique, une méthode de calcul a donc été développée en collaboration avec le LIMSI [1]. Cette méthode de différences finies, que l'on qualifie de semi-inverse, permet de définir la forme des AV. D. (en fait leur ligne de cambrure), afin d'obtenir une prérotation uniforme à l'entrée des directrices. Ces travaux théoriques [1] sont restés, à la connaissance des auteurs, les seuls dans ce domaine jusqu'à une date récente où une méthode d'éléments finis pour résoudre le problème direct a été proposée [2].

Si l'on considère que l'écoulement dans la bâche peut-être assimilé à celui d'un fluide parfait quasitridimensionnel incompressible, il est défini par la fonction de courant. Le fluide étant supposé non visqueux et irrotationnel : la fonction de courant est alors régie par une équation de Poisson [3]. Cette équation est résolue par une méthode semi-inverse [1]. Une première étape consiste à déterminer l'écoulement đans la bâche sans avant-directrice. En imposant une vitesse đébitante homogène sur le cercle délimitant les bords de fuites des AV. D. on a une condition de type Dirichlet sur cette frontière. La résolution numérique du champ détermine l'inclinaison des lignes de courant à la frontière. En raccordant ce champ à celui d'un puits tour. billon (champ de vitesse homogène demandé) on observe une discontinuité de l'écoulement. Cette discontinuité représente une couche de tourbillon de densité non uniforme.

L'étape suivante consiste à transporter cette répartition tourbillonnaire à l'emplacement présumé des AV.D. et à mener le calcul en tenant compte de cette circulation. Le maillage est modifié de manière à s'aligner sur les lignes de courant. A la convergence, les AV. D. sont confondues avec une portion de lignes de courant porteuses d'une densité adéquate de tourbillon.

\section{Formulation théorique}

La transformation suivante ramène le plan physique $(m, \theta)$ de la figure 1 au plan de calcul $(x, y)$ de la figure 2.

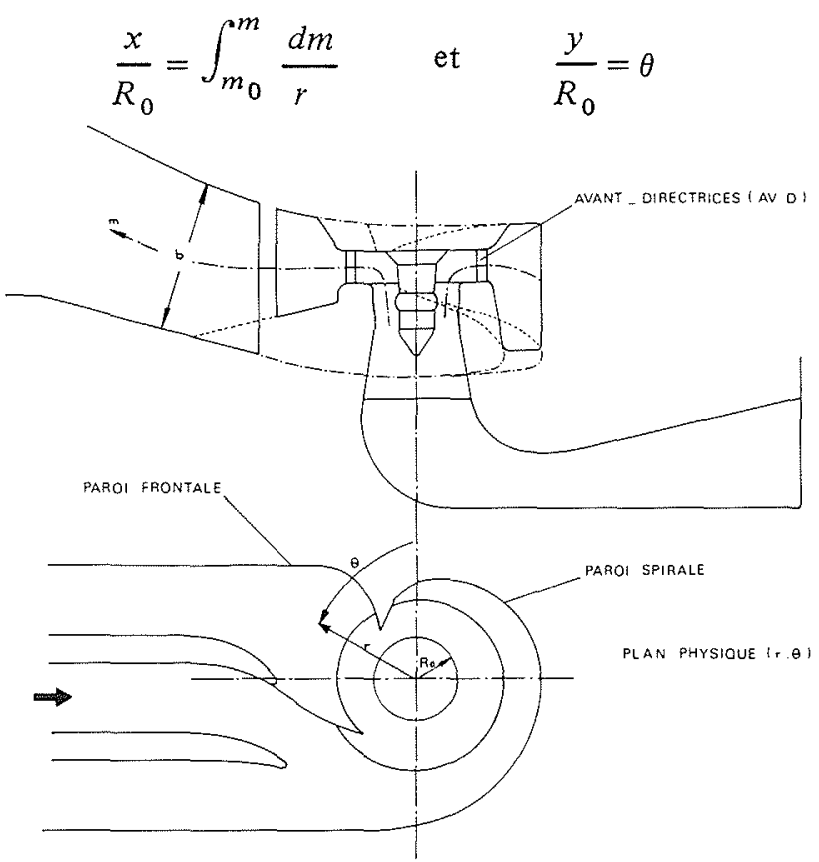

Figure 1 - Géométrie caractéristique d'une bâche.

LA HOUILLE BLANCHE/N ${ }^{\circ} 7 / 8-1982$ 


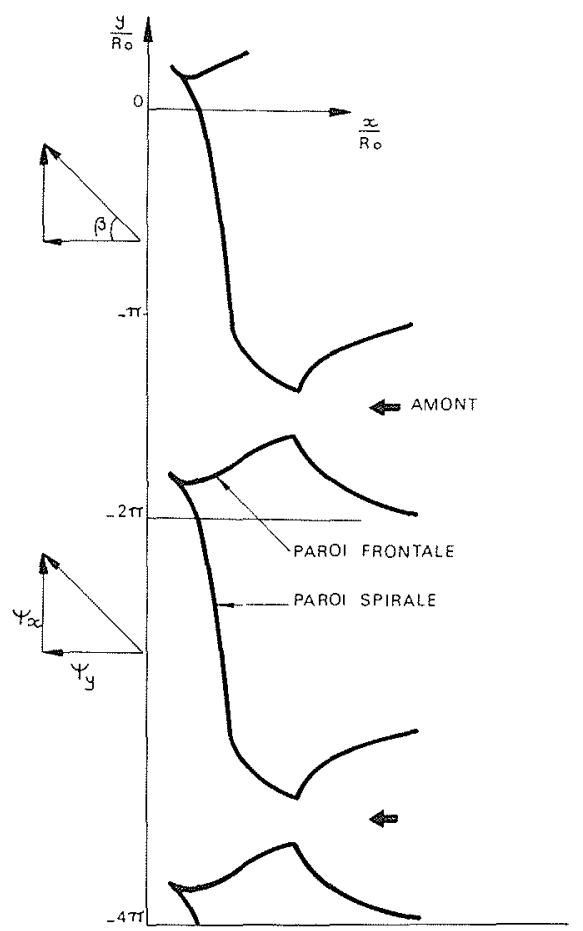

Figure 2 - Grille de profils ouverts dans le plan transformé $(x, y)$.

$$
\text { et } \quad \text { si } m=r \quad \frac{x}{R_{0}}=1 n \frac{r}{R_{0}}
$$

où $R_{0}$ est le rayon de la turbine.

Soit $b$ une loi d'épaisseur, on peut écrire :

$$
\begin{aligned}
d s^{2} & =d m^{2}+r^{2} d \theta^{2}+b^{2} d v^{2} \\
& =\frac{r^{2}}{R_{0}^{2}}\left(d x^{2}+d y^{2}\right)+b^{2} d v^{2}
\end{aligned}
$$

En prenant comme coordonnées $x_{1}=x, x_{2}=y$ et $x_{3}=\nu$ et comme base $\vec{e}_{1}, \vec{e}_{2}$ et $\vec{e}_{3}$ on a d'après (2)

$$
h_{1}=\frac{r}{R_{0}}, \quad h_{2}=\frac{r}{R_{0}} \quad \text { et } h_{3}=b
$$

D'autre part, les formules classiques du gradient, de la divergence et du rotationnel en coordonnées curvilignes donnent [4].

$$
\begin{aligned}
\nabla \dot{\phi} & =\sum_{i=1}^{3} \frac{\phi, i}{h_{i}} \vec{e}_{i} ; \\
\nabla \cdot \vec{V} & =\frac{1}{h_{1} h_{2} h_{3}} \Sigma\left(V_{i} h_{j} h_{k}\right)_{, i} \\
\nabla_{\Lambda} \vec{V} & =\sum \frac{1}{h_{1} h_{2}}\left[\left(V_{2} h_{2}\right)_{, 1}-\left(V_{1} h_{1}\right)_{, 2}\right] \vec{e}_{3}
\end{aligned}
$$

Si $\vec{V}$ désigne la vitesse telle que $\vec{\nabla}=V_{1} \vec{e}_{1}+V_{2} \vec{e}_{2}$, le fluide étant incompressible implique que $\nabla \vec{V} \stackrel{2}{=} \stackrel{0}{0}$ soit :

$$
\frac{R_{0}}{r^{2} b}\left[\frac{\partial}{\partial x}\left(\frac{b r}{R_{0}} V_{1}\right)+\frac{\partial}{\partial y}\left(\frac{b r}{R_{0}} V_{2}\right)\right]=0
$$

Cette équation est automatiquement satisfaite par l'introduction de la fonction de courant $\Psi$ telle que :

$$
\frac{b r}{R_{0}} V_{1}=\Psi_{y} \quad \frac{b r}{R_{0}} V_{2}=-\Psi_{x}
$$

En utilisant les expressions (3) et le fait que l'écoulement est irrotationnel, on obtient l'équation régissant $\Psi$.

$-\frac{R_{0}}{r^{2} b}\left[\Psi_{x x}+\Psi_{y y}-\left(\Psi_{x} \frac{\partial \ln b}{\partial x}+\Psi_{y} \frac{\partial \ln b}{\partial y}\right)\right]=\tau_{3}$

où $\tau_{3}$ ast la composante du rotationnel suivant la direction $\vec{e}_{3}$.

La figure 2 représente le domaine transformé $D_{1}(x, y)$ du domaine physique $D_{2}(r, \theta)$. Dans $D_{1}$, on crée un maillage constitué par deux familles de courbes $\xi_{1}=$ Cte et $\xi_{2}=$ Cte. La génération de ce maillage est obtenue par la méthode proposée par [5] où les fonctions $\xi_{1}(x, y)$ et $\xi_{2}(x, y)$ sont régies respectivement par l'équation de Laplace :

$$
\nabla^{2} \xi_{1}=0 \text { et } \nabla^{2} \xi_{2}=0
$$

La détermination du maillage dans le plan $D_{1}$ conduit à un problème de champ posé dans le domaine $D_{3}\left(\xi_{1}\right.$, $\left.\xi_{2}\right)$. $D_{1}$ est représenté dans $D_{3}$ par un maillage régulier de $\operatorname{pas} \nabla \xi_{1,2}=1$.

Dans $D_{3}$, les équations (5) deviennent après transformation :

$$
\left.\begin{array}{l}
\alpha x_{\xi_{1} \xi_{1}}-2 \beta x_{\xi_{1} \xi_{2}}+\gamma x_{\xi_{2} \xi_{2}}=0 \\
\alpha y_{\xi_{1} \xi_{1}}-2 \beta y_{\xi_{1} \xi_{2}}+\gamma y_{\xi_{2} \xi_{2}}=0
\end{array}\right\}
$$

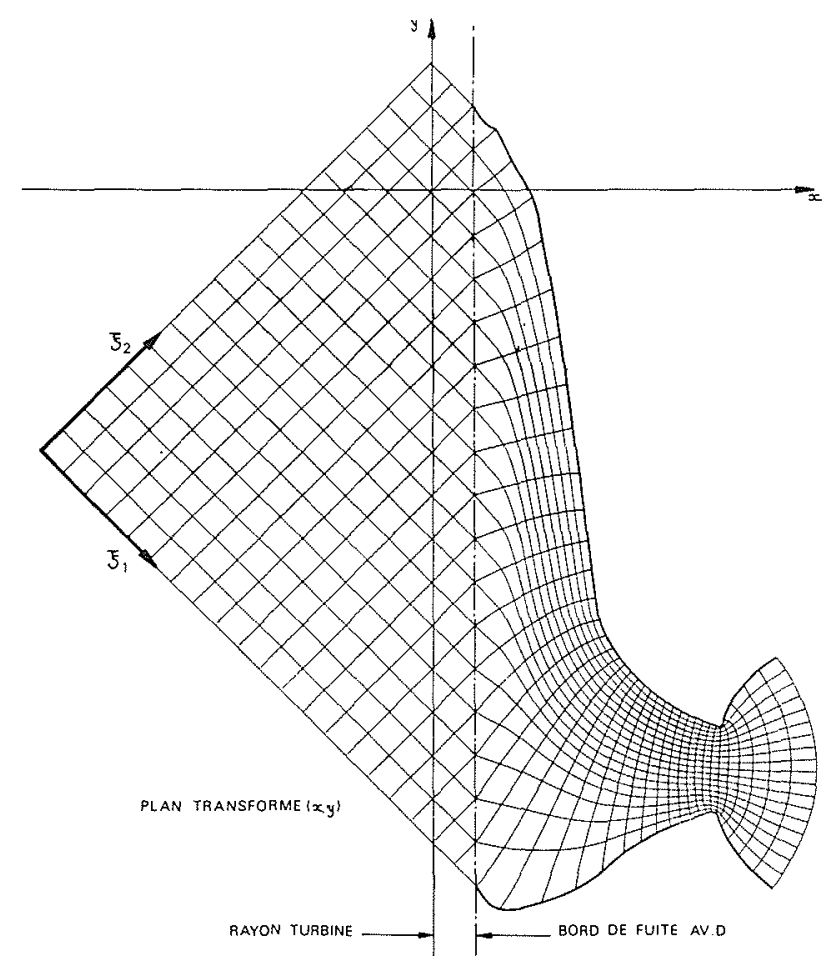

Figure 3 - Maillage sans aube dans le plan transformé $\beta=45^{\circ}$. 
où

$$
\begin{aligned}
& \alpha=x_{\xi_{2}}^{2}+y_{\xi_{2}}^{2} \\
& \beta=x_{\xi_{1}} x_{\xi_{2}}+y_{\xi_{1}} y_{\xi_{2}} \\
& \gamma=x_{\xi_{1}}^{2}+y_{\xi_{1}}^{2}
\end{aligned}
$$

Le système (6) représente les équations régissant $x$ et $y$ dans $D_{3}$. En résolvant ce système par différencesfinies et en utilisant aux frontières une condition du type Dirichlet, on obtient un maillage du type de la figure 3. La transposition de l'équation (4) dans le nouveau domaine de calcul $D_{3}$ s'écrit [1] :

$$
\begin{aligned}
-\frac{1}{b}\left[A \Psi_{\xi_{1} \xi_{1}}+C\right. & \Psi_{\xi_{2} \xi_{2}}+2 B \Psi_{\xi_{1} \xi_{2}} \\
& \left.-D \Psi_{\xi_{1}}-E \Psi_{\xi_{2}}\right] J \delta \xi_{1} \delta \xi_{2}=\Gamma_{i, j}
\end{aligned}
$$

où $\Gamma_{i, j}$ représente la circulation surfacique $\tau_{3} d s$ autour d'un noeud du maillage et $J$ le Jacobien de la transformation.

Les coefficients de l'expression (7) s'écrivent :

$$
\begin{aligned}
A & =\xi_{1, x}^{2}+\xi_{1, y}^{2}=\frac{1}{J^{2}}\left[x_{\xi_{2}}^{2}+y_{\xi_{2}}^{2}\right]=\frac{\alpha}{J^{2}} \\
C & =\xi_{2, x}^{2}+\xi_{2, y}^{2}=\frac{1}{J^{2}}\left[x_{\xi_{1}}^{2}+y_{\xi_{1}}^{2}\right]=\frac{\gamma}{J^{2}} \\
B & =\xi_{1, x} \xi_{2, x}+\xi_{1, y} \xi_{2, y} \\
& =-\frac{1}{J^{2}}\left[x_{\xi_{2}} x_{\xi_{1}}+y_{\xi_{2}} y_{\xi_{1}}\right]=-\frac{\beta}{J^{2}} \\
D & =-\nabla^{2} \xi_{1}+A \frac{\partial L_{n} b}{\partial \xi_{1}}+B \frac{\partial L_{n} b}{\partial \xi_{2}} \\
E & =-\nabla^{2} \xi_{2}+B \frac{\partial L_{n} b}{\partial \xi_{1}}+C \frac{\partial L_{n} b}{\partial \xi_{2}}
\end{aligned}
$$

La formulation des coefficients A, B, C, D et E fait intervenir les expressions suivantes :

et

$$
\begin{gathered}
\partial \xi_{i} / \partial x_{j}(i=1,2 \text { et } j=1,2) \\
\partial^{2} \xi_{k} / \partial x_{i} \partial x_{j}(k=1,2 ; i=1,2 \text { et } j=1,2)
\end{gathered}
$$

qui peuvent être déterminées d'une part par le système de 4 équations.

$$
\sum_{n=1}^{2} \frac{\partial x_{k}}{\partial \xi_{n}} \cdot \frac{\partial \xi_{n}}{\partial x_{j}}=\delta_{k, j}
$$

avec $\delta_{k, j}$ symbole de Kronecker.

et d'autre part, par le système de 6 équations :

$$
\begin{aligned}
\sum_{n=1}^{2} \frac{\partial x_{k}}{\partial \xi_{n}} \cdot \frac{\partial^{2} \xi_{n}}{\partial x_{i} \partial x_{j}} & \\
& =-\sum_{m=1}^{2} \sum_{n=1}^{2} \frac{\partial^{2} \xi_{k}}{\partial \xi_{m} \partial \xi_{n}} \cdot \frac{\partial \xi_{m}}{\partial x_{i}} \cdot \frac{\partial \xi_{n}}{\partial x_{j}}
\end{aligned}
$$

Enfin on montre que les expressions $\nabla^{2} \xi_{1}$ et $\nabla^{2} \xi_{2}$ s'écrivent:

$$
\begin{aligned}
& \nabla^{2} \xi_{1}=\left[-\alpha\left(y_{\xi_{2}} x_{\xi_{1} \xi_{2}}-x_{\xi_{2}} y_{\xi_{2}} y_{\xi_{1} \xi_{1}}\right)-\gamma\right. \\
& \left.\left(y_{\xi_{2}} x_{\xi_{2} \xi_{2}}-x_{\xi_{2}} y_{\xi_{2} \xi_{2}}\right)+2 \beta\left(y_{\xi_{2}} x_{\xi_{1} \xi_{2}}-x_{\xi_{2}} y_{\xi_{1} \xi_{2}}\right)\right] / J^{3} \\
& \nabla^{2} \xi_{2}=\left[\alpha\left(y_{\xi_{1}} x_{\xi_{1} \xi_{1}}-x_{\xi_{1}} y_{\xi_{1} \xi_{1}}\right)+\gamma\right. \\
& \left.\left(y_{\xi_{1}} x_{\xi_{2} \xi_{2}}-x_{\xi_{1}} y_{\xi_{2} \xi_{2}}\right)-2 \beta\left(y_{\xi_{1}} x_{\xi_{1} \xi_{2}}-x_{\xi_{1}} y_{\xi_{1} \xi_{2}}\right)\right] / J^{3}
\end{aligned}
$$

\section{Résolution sans aubes}

La résolution du champ $\Psi$ est effectuée dans le domaine situé en aval du lieu délimitant le bord de fuite des AV. D., la prérotation étant représentée par un champ uniforme qui est engendré par un puitstourbillon placé au centre. La discontinuité de l'écoulement à travers cette ligne fait apparaître une couche de tourbillons ayant une densité non uniforme déterminée par:

$$
\begin{aligned}
\hat{\gamma}=-\frac{R o}{b r}\left[\left(\Psi_{\xi_{1}}^{+} \xi_{1, x}^{+}+\right.\right. & \left.\Psi_{\xi_{2}}^{+} \xi_{2, x}^{+}\right) \\
& \left.-\left(\Psi_{\xi_{1}}^{-} \xi_{1, x}^{-}+\Psi_{\xi_{2}}^{-} \xi_{2, x}^{-}\right)\right](9)
\end{aligned}
$$

où les dérivées sont évaluées par différences finies décentrées à droite ou à gauche respectivement pour le signe $(+)$ et $(-)$.

La circulation $\Gamma_{j}$ pour un intervalle azimutal $y_{j}$, $y_{j+1}$ est donc

$$
\Gamma_{j}=\frac{r}{R_{0}} \int_{y_{j}}^{y_{j}+1} \hat{\gamma} d y
$$

\section{Détermination des avant-directrices}

La circulation obtenue par (10) doit donc être transportée du lieu des bords de fuite sur l'emplacement présumé de l'aube $j$ si l'on considère que $\left(y_{j+1}+y_{j}\right) / 2$ est l'ordonnée azimutale de l'aube numéro $j$.

\subsection{Répartition surfacique}

A ce stade du calcul on discrétise en $N$ segments la répartition de simple couche sur le tracé présumé des aubes, pour l'aube $\mathrm{N}^{\circ} j$ et au noud $(i, j)$ la circulation

$$
\Gamma_{i j}=\frac{\Gamma_{j}}{N-1}
$$

sera introduite comme une répartition surfacique de tourbillon, les nouds du bord d'attaque et de fuite sont traités de façon particulière pour tenir compte de l'adaptation souhaitable du bord d'attaque et de la condition de Kutta-Joukowsky au bord de fuite.

Sur la frontière située à l'aval, une loi linéaire de $\Psi$ est imposée :

$$
\Psi=\Psi_{y} y+K=\frac{b(r V m)}{R_{0}} y+k
$$

Le processus de résolution est itératif, à la convergence du calcul du champ $\Psi$ correspond au degré de 
prérotation souhaité du projet, les lignes $\xi_{2}=$ Cte sont modifiées pour correspondre aux lignes de courant. La mise à jour du maillage est obtenue par interpolation.

\subsection{Répartition linéique de $\Gamma_{i j}$}

La représentation du rotationnel $\Gamma_{i j}$ est maintenant engendrée par une répartition linéique de tourbillon sur l'aube représentée par une portion de ligne $\xi_{2}=\mathrm{Cte}$. La direction tangentielle $\vec{s}$ de celle-ci s'aligne avec le déplacement engendré par la variation $-\delta \xi_{1}$. La répartition linéique de $\Gamma_{i j}$ s'écrit :

$$
\begin{aligned}
\Gamma_{i j} & =\left(V_{s}^{+}-V_{s}^{-}\right) \delta s \\
& =\frac{1}{b}\left[\left(\frac{\beta^{+}}{J^{+}}-\frac{\beta^{-}}{J^{-}}\right) \Psi_{\xi_{1}}-\gamma\left(\frac{\Psi_{\xi_{2}}^{+}}{J^{+}}-\frac{\Psi_{\xi_{2}}^{-}}{J^{-}}\right)\right] \delta \xi_{1}
\end{aligned}
$$

de l'expression (11) on déduit l'équation régissant $\Psi$

$$
\gamma\left(\frac{\Psi_{\xi_{2}}^{+}}{J^{+}}-\frac{\Psi_{\xi_{2}}^{-}}{J^{-}}\right)=\left(\frac{\beta^{+}}{J^{+}}-\frac{\beta^{-}}{J^{-}}\right) \Psi_{\xi_{1}}-b \Gamma_{i j}
$$

Dans cette équation les signes $(+)$ et $(-)$ signifient que les expressions doivent être évaluées respectivement sur les faces supérieures et inférieures des aubes, ce qui conduit à l'utilisation de dérivées décentrées.

\section{Méthode numérique}

Le processus de résolution est une méthode de différences finies du type factorisation approchée (AF1, Approximate Factorisation 1) [6].

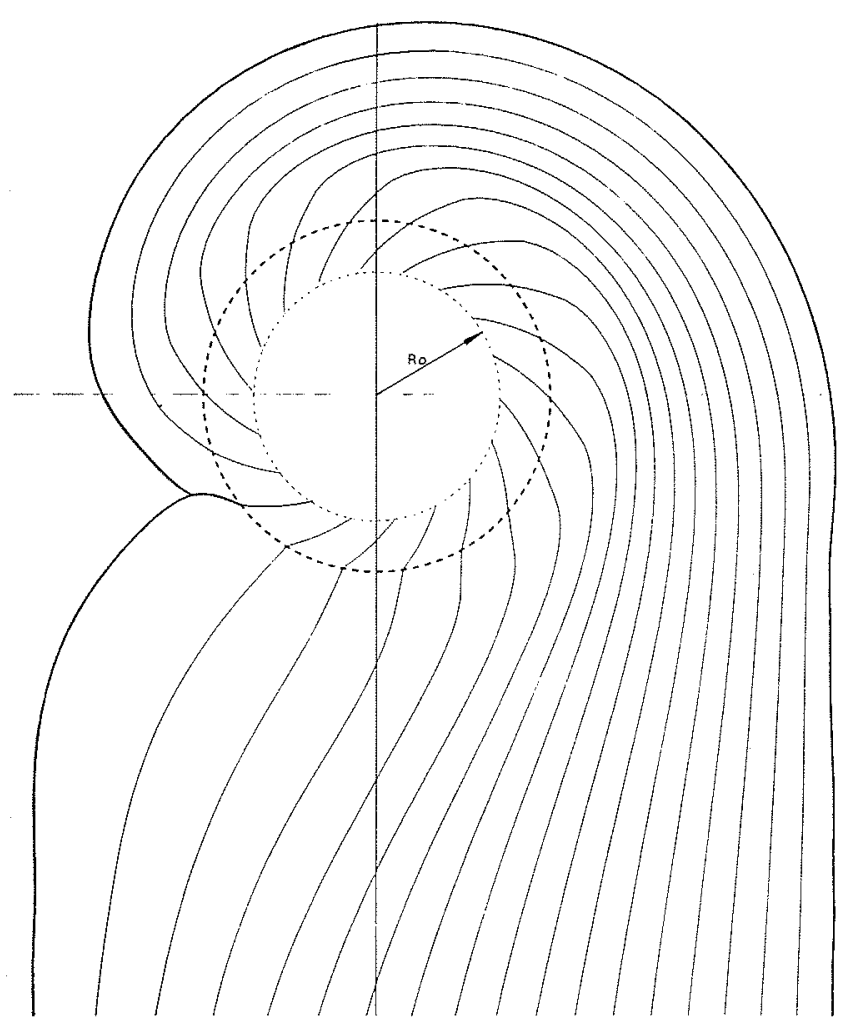

Figure 4 - Lignes de courant sans aube $\beta=45^{\circ}$
Cette méthode converge beaucoup plus rapidement que les méthodes traditionnelles de résolution des équations du second-ordre comme la relaxation (SLOR par exemple). On peut ainsi gagner un facteur 4 sur les temps de calcul.

Cette amélioration de la convergence est à rattacher au processus implicite du calcul suivant les deux directions $\xi_{1}$ et $\xi_{2}$. De plus le calcul ne génère que des systèmes linéaires tridiagonaux à diagonale principale prépondérante permettant ainsi une résolution efficace.

\section{Résultats numériques}

\subsection{Maillage sans aubes}

Comme nous l'avons exposé au paragraphe III la première étape du calcul consiste à déterminer le champ $\Psi$ sans prise en compte des AV. D. Pour une bâche caractéristique d'une turbine Kaplan, les lignes de courant calculées sont représentées en fig. 3 et 4 respectivement pour le plan de calcul et le plan physique. On notera sur la fig. 3 la discontinuité de ces lignes de courant sur le rayon du bord de fuite des AV. D. qui représente la couche de tourbillon définie en (9).

Il est intéressant de comparer la direction des lignes de courant caractérisée par l'angle $\beta$ (par rapport au rayon) telle que le délivre la bâche suivant le type de loi d'épaisseur choisi. Cette comparaison est présentée en fig. 5 où trois lois sont considérées :

$-b=\mathrm{C}^{\text {te }}$

$-b=f(r)$ loi simplifiée adoptée en [1]

$-b=f(r, \theta)$

On notera l'importance de cette loi d'épaisseur pour assurer l'homogénéité de l'angle dans la partie spirale. Malheureusement dans la zone frontale les angles sont notoirement plus faibles et un distributeur même très tolérant en incidence travaillerait dans de mauvaises conditions d'où l'intérêt d'AV. D. convenablement conçues.

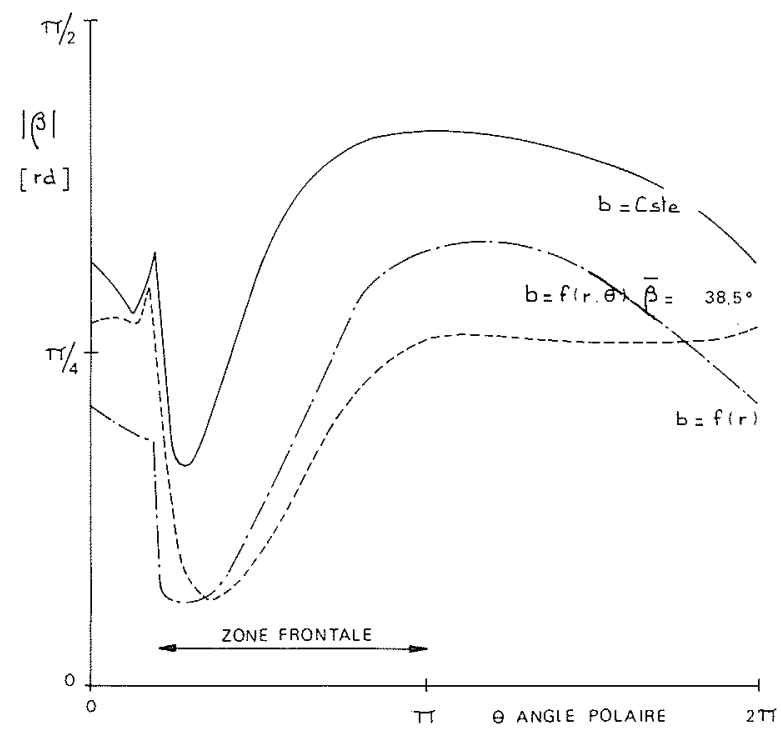

Figure 5 - Influence de la loi d'épaisseur sur l'angle d'injection $\beta$. 


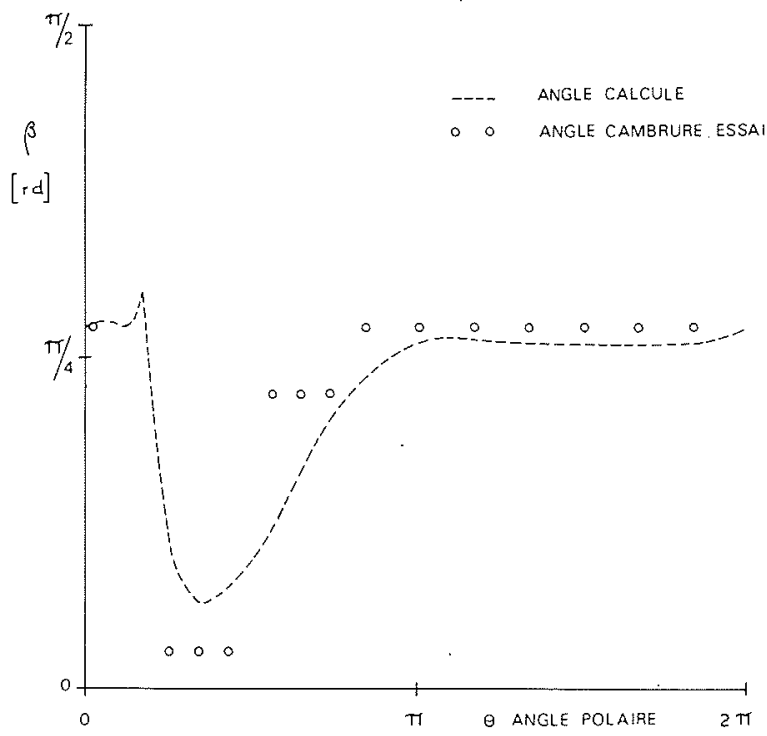

Figure $6 \mathrm{a}$ - Comparaison de l'angle du bord d'attaque des AV.D. avec l'angle calculé.

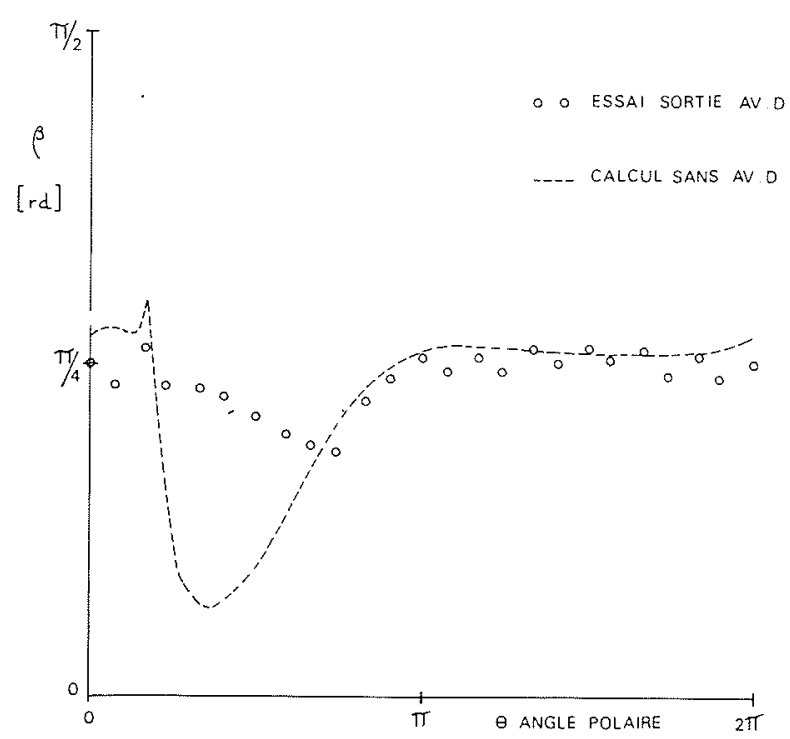

Figure $6 \mathrm{~b}$ - Angle $\beta$ au niveau du cercle de sortie des AV.D.

En fig. $6 \mathrm{a}$, on compare le calcul sans aube pour la loi $b(r, \theta)$ à l'angle donné par la loi de cambrure au bord d'attaque des AV. D. utilisées lors d'une visualisation à l'eau sur modèle réduit. Lors de cet essai on s'est assuré que les AV. D. avaient un bord d'attaque sensiblement adapté. La relativement bonne concordance entre ces deux courbes nous autorise à penser que les lignes de courant calculées sont en bon accord avec la réalité physique. En outre si l'on considère les $A V$. D. qui sont en incidence (zone frontale), il se trouve comme on peut le voir fig. $6 \mathrm{~b}$, que c'est également la zone où l'angle de sortie des AV. D, est le plus faible lors de l'essai.

\subsection{Maillage avec aubes}

A ce stade du calcul intervient un paramètre essentiel qui est l'angle désiré à la sortie des AV. D. Comme nous l'avons vu fig. 5 la bâche fronto-spirale sans avantdistributeur n'est pas capable de délivrer un angle cons- tant. Alors qu'un bon dimensionnement de la partie spirale conduit à une valeur pratiquement constante, ici $\beta=45^{\circ}$, la zone frontale ne donne qu'une très faible valeur de $\beta$ ce qui entraîne qu'en moyenne $\bar{\beta}=38.5^{\circ}$. Or, la charge des AV. D. est essentiellement liée à la valeur de l'angle $\beta$ que l'on désire. Nous présenterons donc une comparaison des résultats obtenus en imposant que l'angle à la sortie des $\mathrm{AV}$. D. prenne les trois valeurs suivantes : $\beta=35.7^{\circ}, 40^{\circ}$ et $45^{\circ}$. Le nombre d'aubes retenu pour ces trois configurations restera figé et égal à 17 .

Dans le plan physique de la bâche on obtient le réseau de ligne de courant présenté en fig. 7 et 8 pour les deux valeurs extrêmes de $\beta$ envisagées précédemment. On observera que l'on considère des aubes sensiblement plus longues dans la zone frontale, ceci afin d'obtenir un pas relatif en conformité avec le niveau de déviation souhaité dans cette partie (jusqu'à $30^{\circ}$ environ). Si l'on ne prenait pas cette précaution on aurait des risques de décollement ce qui invaliderait le calcul.

La circulation portée par chaque AV. D. représente la charge supportée par celle-ci. Nous présentons donc fig. 9 la circulation calculée qui correspond aux trois valeurs de $\beta$. Cette circulation adimensionnelle $\Gamma_{j} b / Q$ (où $\Gamma_{j}$ est donné par (10), b est la hauteur du distributeur et $Q$ est le débit) ne dépend que de la déviation angulaire. Nous pouvons observer une variation quasilinéaire de la charge dans la zone frontale alors qu'elle reste pratiquement constante dans la partie spirale. Nous retrouvons ainsi les résultats obtenus par expérimentation, particulièrement l'utilisation d'AV. D. identiques dans la zone spirale. En outre, la variation importante de $\Gamma$ dans partie frontale montre que c'est dans cette zone que les $\mathrm{AV}$. D. sont critiques et doivent être définies avec le plus grand soin.

$\mathrm{La}$ confrontation des divers calculs fig. 9 amène certaines remarques. $\mathrm{Au}$ vu des résultats on pourrait être tenté de considérer que la solution $\beta=35.7^{\circ}$ est plus avantageuse car elle assure une meilleure répartition de la charge entre zones frontales et spirales. En fait, dans la zone spirale, une telle situation conduit à des aubes décélératrices ce qui n'est pas avantageux et peut poser des problèmes de décollement. La solution $\beta=45^{\circ}$ est plus intéressante, les aubes les plus fortement chargées sont accélératrices, elles n'intéressent qu'une zone azimutale plus réduite où la diminution du pas relatif sera plus facile et moins coûteuse.

Une comparaison quantitative des résultats numériques présentés en [2] et de notre calcul fait l'objet de la figure 10. Nous avons porté la valeur $n \Gamma_{j} / \Gamma$ total, où $\Gamma$ total est la circulation totale délivrée par la bâche et les AV.D. et $n$ le nombre d'aubes. Cette quantité représente en quelque sorte une circulation relative des AV.D. par rapport à la circulation totale. La comparaison bien que quantitative (les deux bâches frontospirales ne sont pas identiques) montre que l'ordre de grandeur des circulations observées est le même ainsi que l'évolution générale de la charge. Par contre la variation de circulation des AV.D. dans la zone spirale observée en [2] tranche avec nos propres calculs, elle pourrait être la conséquence d'une loi d'épaisseur sensiblement différente dans la zone spirale. 


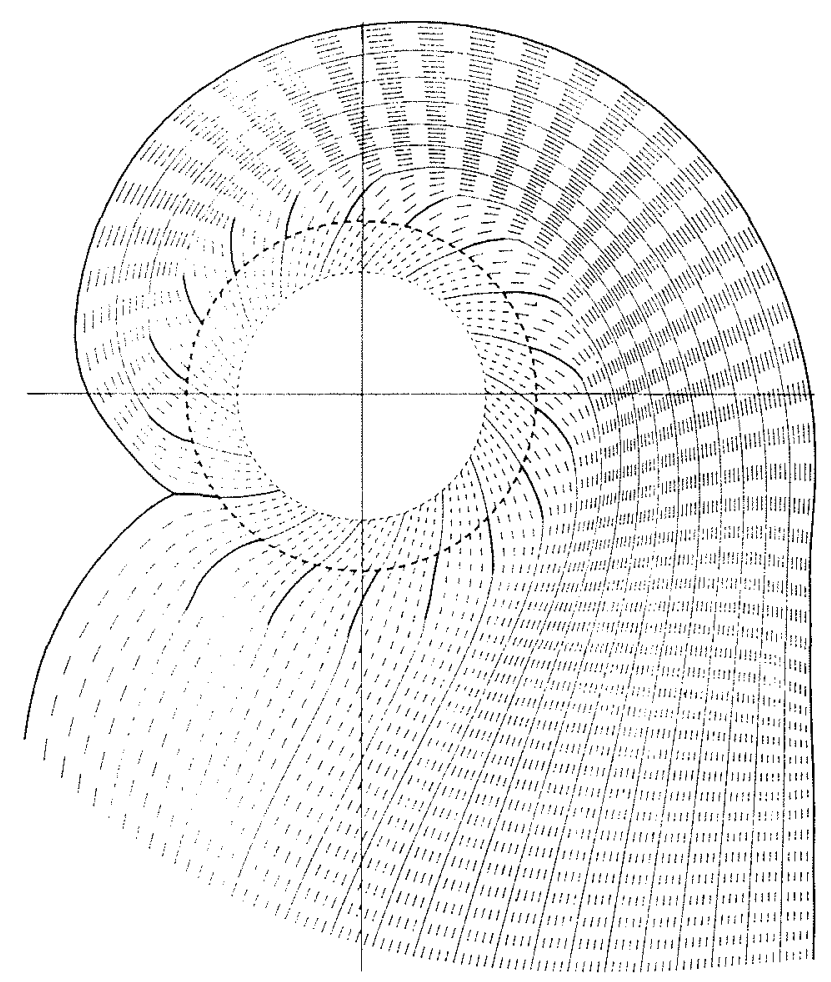

Figure 7 - AV.D. dans le plan phy sique $\beta=35.7^{\circ}$.

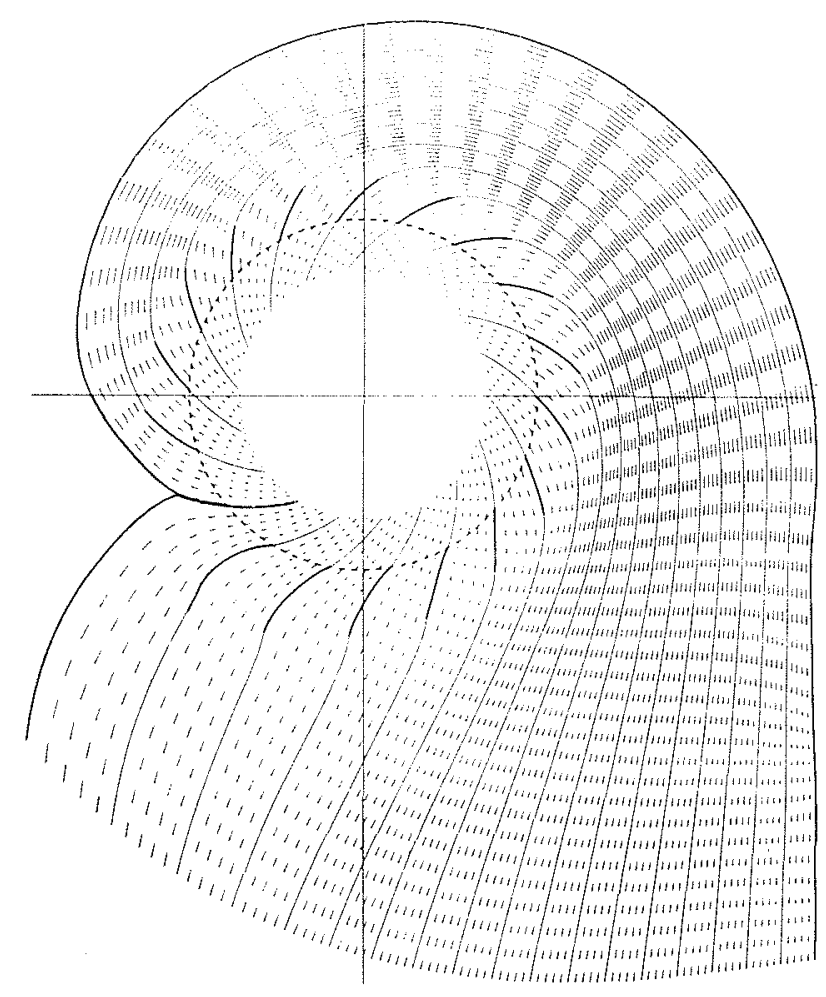

Figure 8 - AV.D. dans le plan physique $\beta=45^{\circ}$.

\subsection{Développements ultérieurs}

Un certain nombre de résultats intéressants ayant été obtenus pour cette bâche une des prochaines étapes de notre recherche consistera en la fabrication des aubes calculées afin de permettre une vérification expérimentale plus approfondie de nos calculs.

Parallèlement à cette étude expérimentale un développement intéressant consistera en la prise en compte de la (où des) pile(s) dans le calcul. En effet, dans notre approche actuelle la ligne moyenne de la pile est définie a posteriori comme portée par une ligne de courant calculée. Ce faisant, elle n'est pas chargée hydrodynamiquement mais risque d'être mal adaptée au génie civil.

D'autres paramètres importants peuvent également être étudiés de manière économique (le temps de calcul d'une configuration n'excède pas l'équivalent de $2 \mathrm{mn}$ de CPU d'un CDC 7600) et conduire à des tracés de bâche mieux adaptés. Citons par exemple, la position du bec de bâche, la hauteur du distributeur, l'utilisation de rampes de formes variées en zone frontale. En outre, cette méthode peut s'appliquer directement aux bâches spirales des turbines Francis et moyennant certaines adaptations devrait pouvoir être utilisée pour la conception des volutes de pompe.

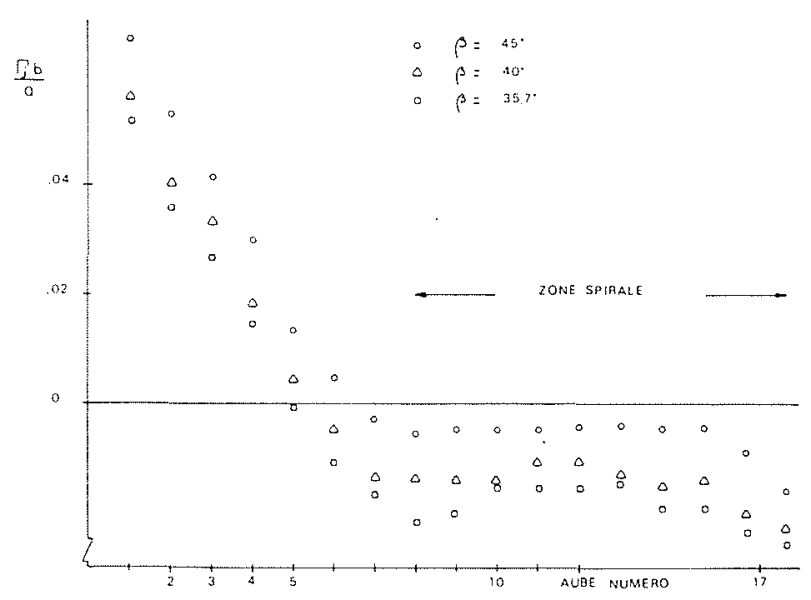

Figure 9 - Circulation adimensionnelle associée à chaque avantdirectrice.

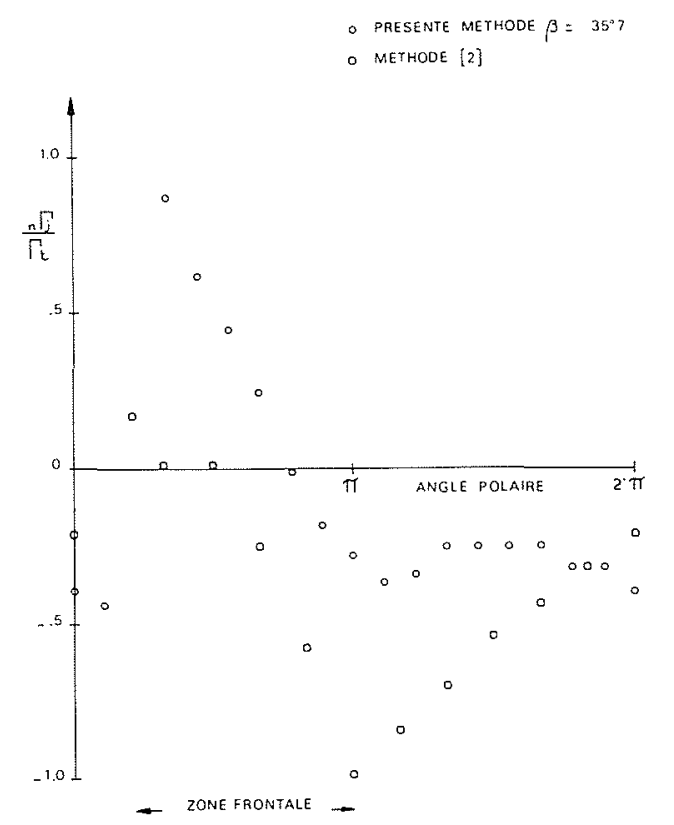

Figure 10 - Comparaison des circulations liées aux AV.D. 


\section{Conclusions}

Une méthode de calcul de l'écoulement dans les bâches fronto-spirales a été présentée. Cette méthode semi-inverse permet de définir la géométrie des avantdirectrices nécessaire à l'obtention d'un champ homogène, de prérotation donnée, à l'entrée du distributeur.

Un certain nombre de résultats numériques ont été analysés et confrontés à des résultats d'essai ou à d'autres calculs lorsque cela était possible.

Cette méthode doit permettre d'étudier les divers paramètres importants de la bâche et, par son analyse fine de l'écoulement, autoriser une optimisation plus rapide de l'amenée des turbines Kaplan.

L'application aux bâches spirales des Francis et aux volutes des pompes est envisageable sans grosse difficulté.

\section{Références}

[1] LUU T.S., VINEY B. et CORNIGLION J. - Conception optimale de l'alimentation latérale d'une turbomachine. $18^{\mathrm{e}}$ colloque d'Aérodynamique appliquée, Poitiers, 1820 Novembre 1981.

[2] WU ERH-RONG. - Finite element modeling and flow prediction for a spiral case. IAHR-Operating problems of pump stations and power plants. Amsterdam, September 1982.

[3] WU C.H. - A general theory of three - dimensional flow in subsonic and supersonic turbomachine of axial, radial and mixed flow types. NACA technical note 2604 , Washington, D.C., January 1952.

[4] GERMAIN P. - Mécanique des milieux continus, Masson, 1962.

[5] THOMSON J.F., THAMES F.C. and MARTIN C.M. Automatic numerical generation of body fitted curvilinear coordinate system for field containing any number of arbitrary two-dimensional bodies. J. of Comp. Physics, vol 15, 1974, pp. 299-319.

[6] BAKER, TIMONTHY J. - Potentiel flow calculation by the approximative factorisation method. J. of comp. Physics, vol. 42, July 1981, pp. 1-19.

\section{Discussion}

Président $:$ R. BALLAY

A la demande de $\mathrm{M}$. LIESS, M. COUSTON précise que les avant directrices comportent 10 noeuds en zone frontale et 5 sur le secteur restant.

Sur intervention du président, M. COUSTON explique qu'il est difficile de mieux guider l'écoulement dans la zone frontale car il serait nécessaire d'ajouter une alimentation supplémentaire en fond de volute, l'épaisseur de la bâche étant déjà telle qu'elle arrive presque au niveau de l'aspirateur.

M. le Président évoque la possibilité d'extrapoler la méthode de calcul d'une volute de pompe ce qui présenterait un intérêt particulier pour traiter les volutes non conventionnelles des pompes đu nucléaire.

M. CASACCI souligne l'intéfêt de pouvoir uniformiser les vitesses pour pouvoir parvenir à une meilleure prévision des spectres des sillages issus des avant-directrices. L'objectif est de garantir la sécurité vibratoire de la structure.

A la demande de M. JERMANN, M. COUSTON précise que la méthode de calcul permet seulement de déterminer la ligne de cambrure des avant-directrices, leur loi de circulation ayant été arbitrairement imposée. D'autre part, leur loi d'épaisseur et leur longueur sont régies par des considérations d'ordre mécanique.

Contrairement à l'objection de M. JERMANN, M. COUSTON estime que si l'épaisseur est répartie symétriquement par rapport à la ligne de cambrure, il n'y aura pas de modification sensible des angles calculés à l'aval des avant-directrices. 


\section{Abstract \\ A model of the flow in frontospiral tanks}

Up to now the hydraulic conception of the spiral casing used in low head KAPLAN turbines was solved by experimental tests. These approach is undoubtedly time and money consuming. As 20 to $30 \%$ of the losses are located in front of the runner it is very important to optimize the casing and the stay-vanes to insure a correct incoming flow to the wicketgates.

The main objective of this work was thus to develop a theoretical approach of the flow in order to get an homogeneous peripheral velocity distribution at the exit of the stayvanes.

The method is based on the solution of a flow field problem. Making use of the following assumptions, inviscid, irrotationnal and quasi-3D flow the governing equation based on the stream function is of the Poisson type. Using a body fitted coordinate system the second order equation is discretised by finite differences. The numerical solution is than carried using an Alternating Direction Implicit technique known as Approximate Factorisation 1
The computation starts without stay-vanes imposing uniform flow rate at the exit of the casing. This Diricklet boundary condition leads to a solution where the expected uniform prewhirl is not fullfiled. A finite number of stay vanes are than introduced in the field in order to get the correct flow deflection. At the mesh points located on the presumed blade location a distribution of bound vortex is applied, the vorticity is updated until the given prewhirl condition is met. However at the same time the nodal value of the stream function is enforced at the trailing edge of the stay-vanes to maintain the constant peripheral flow rate and the blade position is changed iteratively in order to correspond with a streamline.

At convergence the computation provides the shape and the load of each stay-vane required to obtain the prescribe uniform flow at the wicket-gates inlet. Numerical solutions are presented for a typical KAPLAN casing and compared with available experimental and theoretical results. 